\title{
Construction of Multimedia Teaching Resource Management Evaluation Model in the Big Data Environment
}

\author{
https://doi.org/10.3991/ijet.v13i03.8365 \\ Wei Bian \\ Xijing University, Xi'an, Shaanxi, China \\ bianwei@xijing.edu.cn
}

\begin{abstract}
In view of the deficiencies in the studies of the multi-media teaching resource management method and resource management evaluation model, a multi-media teaching resource management evaluation model in the big data environment is proposed in this paper based on the existing work. The teaching resource management evaluation method of the proposed model is further studied based on the elaboration of the component elements of the model. Guided by the multimedia teaching resource management evaluation model, the construction method for the multimedia teaching resource management evaluation model is studied in the open big data environment. And the multimedia remote teaching data of 360 encyclopedia and news web pages are used as the basis for experimental verification. The results show that the proposed model and method can effectively evaluate the multimedia teaching resource management.
\end{abstract}

Keywords-Big Data Environment; Multimedia Teaching; Resource Management; Remote Teaching

\section{Introduction}

The information society has entered the era of big data. Various types of applications in the multimedia teaching are emerging in an endless stream. Multimedia teaching behavior is increasingly driven by intensive resource management [1]. Resource management is the basis of human intelligence activities. For the computer to use the multimedia teaching resource management, the resource management needs to be structured, so that the computer can have sufficient teaching resources to perform reasoning and calculation [2-3]. Hence the system based on resource management can have intelligence. The traditional teaching resource management is shown mainly in the form of face to face teaching. These traditional teaching resources contain only the basic teaching information [4], making it difficult to provide intensive resource management support for in-depth processing of texts. Subsequently, the concept of ontology was introduced into the field of artificial intelligence, which was then expanded, studied in the field of information science, and used as resource management evaluation model to carry the teaching resources. Early ontology construction was 
time-consuming and labor-intensive. The resource management structure was relatively single, lack of expansion mechanism, and difficult to update dynamically [5]. Later, although ontology was improved in the construction process and resource management structure, it was still static with weak scalability in general and could hardly adapt to the real-time requirements of depth information processing for the resource management in the era of big data [6].

In recent years, the construction method for multimedia teaching resource management model has been widely recognized. In particular, with the full implementation of Linking Open Data and other projects, there is a surge in the number of multimedia teaching resources. In this context, the multimedia teaching resource management evaluation model in the big data environment has gradually shown its value [7]. In view of the deficiencies in the studies of multimedia teaching resource construction method and resource management evaluation model at present, a multi-media teaching resource management evaluation model is first proposed in this paper based on the ontology and hypergraph theory [8-10]. Guided by the model, the construction method for the multimedia teaching resource management evaluation model is studied in the open big data environment. And the multimedia remote teaching data of 360 encyclopedia and news web pages are used as the basis for experimental verification. The results show that the proposed model and method can effectively evaluate the multimedia teaching resource management.

\section{$2 \quad$ Related Word}

To overcome the deficiencies of the traditional model construction, multimedia teaching resources based on the big data have been widely accepted and applied, which mainly extract the concepts and examples automatically from multimedia teaching online encyclopedia data to expand the scale of resource management database. In addition, search engine companies at home and abroad have also constructed resource management maps based on the big data environment, such as Google's Knowledge Graph, Microsoft's Probase, Baidu's Zhixin, Sogou's Zhilifang, etc. Among them, Microsoft's Probase automatically constructed an ontology resource management database consisting more than 2.6 million concepts and 10 million level instances mainly through iterative learning and analysis on over 1.6 billion web pages and query logs of Bing search engine for about two years. In addition, Google's Knowledge Graph multilingual version is expanded based on the Freebase resource management database. Currently, the resource management system contains more than 500 million entries, and over ten billion factual relations between different objects.

In general, there are relatively more studies on the information extraction for various teaching resources in the current big data environment, but relatively few studies on how to combine these data with the multimedia teaching resource management evaluation model effectively. In addition, some of the large-scale multimedia teaching resources constructed are for internal applications only. The interface and related technologies are not open. Combined with the related studies, it is proposed in this 
paper that teaching resource management problem with n-ary relations should be solved from the model level using the big data for the resource management evaluation model. Meanwhile, the computing problem is raised to the model level. Open and computable multimedia teaching resource management evaluation can be constructed through computing operators implementing various kinds of computations.

\section{Multimedia Teaching Resource Management Analysis}

\subsection{Multimedia Teaching Resource Management}

In this paper, the multimedia teaching resource management in the big data environment is studied. It is a set composed of the resource management nodes and the resource management correlations, in which the resource management nodes generally represent the storage units of the resource management units consisting of concepts or objects. Resource management correlation can be divided into internal correlation and external correlation. The internal correlation constitutes the resource management individuals, showing the connotation connection of the resource management. The external correlation is the extension connection between the resource management individuals, which constitute various link relations of the multimedia teaching resource management. The multimedia teaching resource management has large scale, complicated connection and heterogeneous resource management nodes. It is difficult to fully describe the resource management characteristics in the multimedia teaching world by the general binary multimedia teaching diagram. Therefore, it can be referred to as super multimedia teaching. In this paper, a resource management evaluation model in the big data environment is proposed: $\mathrm{DKN}=(V, E, A, V a l, F, G, O)$.

Resource management node $V$ and resource management correlation $E$. $V=\left\{v_{1}, v_{2}, \mathrm{~L}, v_{n}\right\}, v_{i}$ stands for a simple or complex thing or concept, which is composed of cognitively independent resource management elements. $E=\left\{e_{1}, e_{2}, \mathrm{~L}, e_{m}\right\}$, where the hyperedge $e_{i}$ stands for a simple or complex resource management correlation, which is the correlation relationship between the resource management nodes. Hyperedge can be divided into directed and undirected hyperedge. The directed hyperedge $e_{i}=\left\langle\left(r_{i}\right),\left(\lambda_{i}\right)\right\rangle$ is a sequence pairs, $r_{i}$ is a set of the input variables in $e_{i} . \lambda_{i}$ is a set of the output variables in $e_{i}$. The undirected hyperedge $e_{i}=\left(v_{1}, v_{2}, \mathrm{~L}, v_{k}\right)$ is a set of multivariate undirected edges.

Attribute $A$, attribute value set $\mathrm{Val}$, and attribute value mapping function $F$. $A=A_{V} \cup A_{E}$, which is a non-empty finite set of the resource management nodes and resource management correlation attributes, where $A_{V}$ is the resource management node attribute set, $A_{E}$ is the resource management correlation attribute set. $V a l=V a l_{V} \cup V a l_{E}$, which is the range set of the resource management node attributes 
and resource management correlation attributes. $F=F_{V} \cup F_{E}$, which is the attribute value mapping function set on the resource management nodes and resource management correlations, where $F_{V}: V \times A_{V} \rightarrow V a l_{V}, F_{E}: E \times A_{E} \rightarrow V a l_{E}$.

Resource management mapping function $G . \quad G=\{g \mid g(v)=e\}$, which is the correlation function set on the resource management node $V$, indicating different types of correlation between the objects. Various resource management nodes in the multimedia teaching resource management evaluation are composed of a structured multimedia teaching resource management system in accordance with the required factors, levels, structures and functions, etc. The structured process requires linking the resource management nodes through resource management correlation mapping.

Computation operator $O$. $O$ stands for the computation operator set. The computation operators mainly complete various computing operations for the multimedia teaching resource management. An operator acts on an input to achieve the mapping from one multimedia teaching resource management space to another multimedia teaching resource management space (or itself). Figure 1 shows the computation operator model based on the external information input.

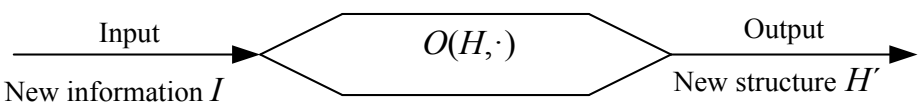

Fig. 1. Computation operator model

The computation operator model based on the external information input can be expressed as: $H^{\prime} \cong O(H, \bullet) I$, in which, $O(H, \bullet)$ is the computation operator, $I$ is the new input information, $H$ is the existing multimedia teaching resource management diagram or diagram structure, $H^{\prime}$ is the new multimedia teaching resource management diagram or sub diagram structure output after the operator computation. The output new structure $\mathrm{H}^{\prime}$ is the result generated by inputting the external information $I$ on the existing structure $H$ after the computation of operator $O(H, \bullet)$. Table 1 provides some common operator examples for multimedia teaching resource management evaluation.

Table 1. Common operator examples for multimedia teaching resource management evaluati-

on

\begin{tabular}{|l|l|l|}
\hline \multicolumn{1}{|c|}{ Operator name } & Formal description & \multicolumn{1}{c|}{ Meaning explanation } \\
\hline Node addition & add_vertex(vi,Ej) & Node addition \\
\hline Attribute clustering & lustering(V,A) & The clustering based on attribute \\
\hline $\begin{array}{l}\text { Solve the hyper-path between } \\
\text { two nodes }\end{array}$ & path(vi,vj,h) & The possible hyper-path between the nodes \\
\hline $\begin{array}{l}\text { Solve the shortest hyper-path } \\
\text { between two nodes }\end{array}$ & hort_path(vi,vj,h) & The shortest path between the nodes \\
\hline Calculate the number of paths & calculate_path(vi,vj) & The possible number of paths between the nodes \\
\hline
\end{tabular}




\subsection{Definition of Multimedia Teaching Resource Management Evaluation}

The multimedia teaching resource management evaluation model studied in this paper is a multimedia teaching model defined by hypergraph. Therefore, such multimedia teaching resource management evaluation is mainly shown in the teaching resource management evaluation for the nodes and edges in the hypergraph. To achieve structural representation of the teaching resources in the multimedia teaching resource management evaluation, the teaching resource management evaluation structure is provided in the multimedia teaching resource management evaluation model.

Representation of the nodes. Node represents a simple or complex object or concept. For the ambiguity in natural language that one evaluation may express a number of different concepts, the nodes we defined mainly include two categories: monosemous nodes and polysemous nodes.

$$
\text { Node }=\left\{(\text { Node }-I d, \text { Node }- \text { Title }), \text { Meaning }- \text { Number },\left[\text { Node }_{1}, \text { Node }_{n}\right]\right\},
$$

In which, when Meaning-Numder $=1$, the node is a monosemous node, Node $=\{($ Node $-I d$, Node - Title $)\}$; otherwise, the node is a polysemous node, Meaning - Numder $=n$, Node $_{i}=\left\{\left(\right.\right.$ Node $-I d_{i}$, Node Title $\left.\left._{i}\right)\right\}$.

Example 1. Monosemous term node.

The People 's Republic of China

$=$ Node $=\{($ Node $-I D=1279256$, Node - Title $=$ The People 's Republic of China $)\}$..

Example 2. Polysemous term node.

Faye Wong

$=\{($ Node $-I D=1279256$, Node - Title $=$ Faye Wong $), 6$,

$($ Node $-I D=3286932-1$, Node - Title $=$ Queen of pop music $)$,

(Node $-I D=3286932-2$, Node - Title $=$ Deputy secretary of the Guangyuan municipal Party committee) ,

(Node $-I D=3286932-3$, Node - Title $=$ Professor at Datong University),L $\}$

Representation of the edges. Edge stands for the correlation relationship between concept nodes. These relationships mainly include the binary relations and the n-ary relations. The relations can be directed or undirected. Generally there are three basic types of edges:

1. Identical correlation edge, which is mainly manifested as the inheritance between concept nodes. The equivalence of nodes is the special case of identical correlation.

2. Subordinate correlation edge, which is mainly manifested as the attribute relations, classification relations and inclusion relations, etc. between resource management nodes;

3. Relevance correlation edge, which is outside the identical and subordinate correlation edges. There is interaction correlation between concept nodes, which is mainly 
manifested as various relations of family, work, etc. It allows the concept nodes to form correlation multimedia teaching in the horizontal direction.

$$
\begin{aligned}
\text { Eage }=\{ & \left(E-\text { Type } E-\text { Direction }, E-\text { Intensity }_{1}\right), \mathrm{L}, \\
& \left.\left(E-\text { Type }_{n}, E-\text { Direction }_{n}, E-\text { Intensity }_{n}\right)\right\}
\end{aligned}
$$

In which, E-Type stands for the type of edge. According to different types of edges, edge mapping function is used for processing. $E$-Direction indicates that the edge is directed or undirected. E-Intensity stands for the edge strength, which value is given by custom definition or relation strength calculation. For example, the attribute edge of concept node:

$$
\begin{aligned}
& E-\text { Type }_{1}=\{\text { name }=\text { Attribute, } \text { type }=\text { Value } \mid \text { Object }, \text { Value } \\
& \text { (attribute-name; attribute-Value) } \mid \text { Object }(\text { attribute-name;Node-Id })\} \\
& \text { Direction }_{1}\{\text { type }=\text { direction }\} ; E-\text { Intensity }_{1}\{\text { value }=\text { user }- \text { defined }\} \text {. }
\end{aligned}
$$

\section{Construction of Multimedia Teaching Resource Management Evaluation Model}

\subsection{Model Construction Method}

The concept and its attributes and relationships are the semantic basis for constructing the multimedia teaching resource management evaluation. In this paper, the attribute of concept is treated as a kind of specific relationship. Therefore, the construction of multimedia teaching resource management evaluation mainly faces two issues: the correlations between concept node extractions, which are introduced in the following section.

Concept node is the main element of multimedia teaching resource management evaluation and the carrier of resource management. The extraction of concept node mainly starts with two kinds of data resources: online multimedia teaching encyclopedia and other web page resources.

Extraction of concept nodes in the online multimedia teaching encyclopedia. In the online multimedia teaching encyclopedia, the extraction of concept nodes is relatively simple. The entries in the encyclopedia are refined and edited at the time of their creation to stand for certain meaning. Therefore, it can usually be regarded as a concept. And each entry has a corresponding explanation page. Hence, the extraction of concept nodes mainly starts with the entry name with explanation page; at the same time, monosemous nodes and polysemous nodes need to be distinguished. The methods for extracting concept nodes in different online multimedia teaching encyclopedia are different. Studies show that there are mainly two processing methods for polysemous concepts in the current main open multimedia teaching encyclopedia: one has 
specialized disambiguation page, such as Wikipedia and Baidu Encyclopedia, etc. The other does not have special disambiguation page, but there is special polysemous term tag added at the head of the explanation page for distinction, such as the Interactive Encyclopedia and 360 Encyclopedia, etc. No matter which processing method is adopted, the final extraction results can be mapped to the node representation method described above by downloading and packaging the corpus data (Wikipedia) or web page source data (Baidu Encyclopedia, etc.), and then parsing and processing.

Extraction of concept nodes in the other web resources. The conceptual nodes in the multimedia teaching resource management evaluation are mainly extracted from the online multimedia teaching encyclopedia; while for other web resources, some unexpected topics or event news web pages related to the practical applications are considered. Some concept nodes extracted from these news web pages already exist in the multimedia teaching encyclopedia, and those not existing can be supplemented to the multimedia teaching resource management evaluation to jointly provide teaching resource support for practical applications. In this paper, the concept node extraction method based on the combination of improved mutual information and information entropy is proposed according to the features of these new web pages.

In the statistical language model, mutual information can be used to quantify the closeness of correlation between strings. If a string set is a complete synthetic word, each component of the synthetic word has relatively high correlation coefficient, and the coefficient is the calculated mutual information value. Let the string set be $S=t_{1} t_{2} \mathrm{~L} t_{i}$, in which $S$ stands for a certain segment of the string set after being split, $t_{i}$ stands for a sequence in the string set $S$ after the face-to-face mechanical segmentation. $M I_{S}$ is used to represent the mutual information value between various sequences in the $S$. The mutual information has a number of computational expressions. In this paper, the simplified mutual information expression is adopted.

$$
M I_{S}=\frac{P\left(t_{1} t_{2} \mathrm{~L} t_{i}\right)}{\sum_{i} P\left(t_{i}\right)-P\left(t_{1} t_{2} \mathrm{~L} t_{i}\right)} \approx \frac{\frac{1}{L} f\left(t_{1} t_{2} \mathrm{~L} t_{i}\right)}{\frac{1}{L} \sum_{i} f\left(t_{i}\right)-\frac{1}{L} f\left(t_{1} t_{2} \mathrm{~L} t_{i}\right)}=\frac{f\left(t_{1} t_{2} \mathrm{~L} t_{i}\right)}{\sum_{i} f\left(t_{i}\right)-f\left(t_{1} t_{2} \mathrm{~L} t_{i}\right)},
$$

In which, $P($.$) stands for the probability, f($.$) stands for the frequency, L$ stands for the total number of sequences. The greater the $M I_{S}$, the closer the correlation between $t_{1} t_{2} \mathrm{~L} t_{i}$, and the more likely $S$ being a complete synthetic word. For a given threshold $\gamma$, if $\left.M I_{S}\right\rangle \gamma, S$ can be used as a candidate synthetic word.

Experiments show that the traditional mutual information calculation method is relatively more suitable for calculating the closeness between 2-gram. For the calculation of more than 2-gram, the calculation result of equation (1) is not good because in the synthetic word composed of polywords, the mutual information calculated value is weakened by the frequency of the occurrence of a single word in it. Hence it is necessary to properly compensate for the calculated value of the polyword terms. In this paper, it is proposed that the compensation value $N_{i}$ (where $i$ stands for the num- 
ber of synthetic words in $S$ ) should be added based on the original calculation of $M I_{S}$. The calculation formula for the synthetic word mutual information of polywords after improvement is as follows.

$$
M I_{S} \approx \frac{f\left(t_{1} t_{2} \mathrm{~L} t_{i}\right)}{\sum_{i} f\left(t_{i}\right)-f\left(t_{1} t_{2} \mathrm{~L} t_{i}\right)} \times N_{i},
$$

$N_{i}$ stands for the parameter related to the number of sequences in the synthetic word. Based on experimental verification, compensation value $N_{i}=i 1 b i$ is set in this paper. The results show that the method of adding the compensation value can greatly improve the recall of synthetic words in the Chinese domain with polywords.

In information theory, information entropy is a measure of uncertain information. In this paper, information entropy is used to evaluate whether a string is a complete word. The principle is that if a string forms a complete word, it is independent of the context where it is located, that is, the greater the changes in its left and right nearest neighborhood, the greater the left and right information entropy. Let any string $S=t_{1} t_{2} \mathrm{~L} t_{i}, t_{1}$ is referred to as the left nearest neighbor of $t_{2}$, and $t_{2}$ is the right nearest neighbor of $t_{1}$. If a string is a word, it will appear a number of times in the text, and its left and right nearest neighbors are often changing, i.e., it exists independently of its left and right nearest neighbors. In this paper, information entropy is used to calculate the left and right nearest neighbors of the strings. The information entropy can be divided into left information entropy and right information entropy. Let the string be $S, L$ be the left nearest neighbor set of $S, R$ be the right nearest neighbor set of $S$, then the calculation expression for the left information entropy $H_{l}(S)$ of $S$ is as follows:

$$
H_{l}(S)=\sum_{\alpha \in L} P(\alpha S) 1 b(1 / P(\alpha S))
$$

In which, $P(\alpha S) \approx f(\alpha S) / \sum_{\beta \in L} f(\beta S)_{\text {stands for the probability of the occurrence }}$ of $\alpha$ in $L, f(\alpha S)$ stands for the frequency of the occurrence of $\alpha$. The right dependent $H_{r}(S)$ expression of $S$ is as follows:

$$
H_{r}(S)=\sum_{\beta \in R} P(S \beta) 1 b(1 / P(S \beta))
$$

For a given threshold $t$, if $\left.H_{l}(S)\right\rangle t$ and $\left.H_{r}(S)\right\rangle t, S$ is considered as a complete synthetic word.

In the big data context, concepts in the specific fields of big data environment emerge in an endless stream. Sometimes it is difficult to achieve full coverage and rapid resource management update even in online multi-media encyclopedia. Based on the acquisition of a large number of news text corpus, the concept node extraction 
method that combines mutual information and information entropy can quickly and accurately extract the concept words in news corpus, as effective supplement to the multimedia teaching encyclopedia extraction results.

The correlation relation reflects the connotation and extension resource management of the concept node, which is the presenter of resource management. In the extraction of correlation relationships, mainly two types of extraction methods are considered: deterministic relationship extraction and fuzzy relationship extraction. The deterministic relationship mainly refers to the relationship type that can be clearly expressed, such as the synonymous relationship, attribute relationship, family relationship, geographic location, organizational affiliation, working relationship, etc. For online multimedia teaching encyclopedia, information is organized according to a predefined set of structures. Therefore, when correlation relationships are extracted, some structured information organization methods can be fully used. (1) Extraction of synonymous relationship: The redirection page in the encyclopedia corpus does not contain the specific explanation content, but only points to the explanation page with the same topic as the current page through the redirect link. This is different teaching resource management for the same concept. The extraction of synonymous relationship can be implemented through the analysis of redirect page; (2) Extraction of attribute relationship: Multimedia teaching encyclopedia uses the information box as a kind of concept node attribute organization method. By analyzing the page information box combined with some simple rules, the attribute relationship of partial concept nodes can be extracted; (3) Extraction of open classification relationship: In the concept node content explanation page, some lexical tags are usually used to classify the concept. We refer to such relationship as open classification relationship, which is represented by undirected hyperedge as a set.

In addition, the extraction of deterministic relationship can also be achieved through some specific language patterns, such as the extraction of synonymous relationship using "Alias", "Original name", "Short name", etc. The attribute relationship of the "Date of birth" can be extracted using "Born in", "Born", "Birth" and other adjacent date information. And the attribute relationship of the "Birthplace" can be extracted using the adjacent geographical location information. These language templates can act on the text content of the encyclopedia entry, and can also act on the news page corpus, so as to achieve the extraction of partial deterministic relationship.

The fuzzy relationship mainly refers to the type of relationship between concept nodes which is difficult to define. But there are certain links between them, and the degree of correlation can be very close. It is usually manifested in the co-occurrence relationship in the texts. For example, there is a large amount of internal link information in the content page of encyclopedia entries. Such link information shows certain strength of correlation relationship existing between the concept nodes. For such relation processing, we mainly calculate the co-occurrence frequency to obtain the numerical intensity representation of the correlation relationship. For the internal link concept nodes in the encyclopedia, the strength of co-occurrence is calculated based on the frequency of appearance of other nodes with page as the unit, so as to obtain the correlation strength between the concept nodes explained in this page and the other nodes. In addition, this kind of correlation is directional, i.e., the correlation 
strength of "Beijing Forbidden City $\rightarrow$ Qing Dynasty" and "Qing Dynasty $\rightarrow$ Beijing Forbidden City" may not be the same, and the strength values are calculated from the respective content pages. For the concept nodes that appear in the news web pages, sentence is used as the co-occurrence unit to calculate the mutual information value between nodes, so as to obtain the value of correlation strength. The final correlation strength value between concept nodes is obtained by weighting the two numerical values obtained from the encyclopedic text and the news corpus. And the correlation strength between some concept nodes may not be high. However, as the occurrence of some events show very strong correlation, this is the resource management information required for practical application, which also shows the rationality of the calculation method.

\subsection{Experiment}

In this paper, Chinese 360 encyclopedia and the news report web pages of "2013 Syria's chemical weapon crisis" are selected as the experimental data set to carry out the experiment on constructing multimedia teaching resource management evaluation.

Chinese 360 encyclopedia information processing. 360 encyclopedia is a multimedia teaching encyclopedia launched in January 2013. As of the end of 2013, $5,732,582$ web page source files had been crawled with the total size of about 100GB. And the extraction process is as follows:

4. Processing of polysemous nodes. There is no specific page for disambiguation in 360 encyclopedia, and polysemous node content is included in the same interpretation page, with clear tags for distinction. The unified expression of polysemous term is extracted from the web page source code, such as the "Forbidden City"; it is convenient to extract the entries with different meaning from the polysemous node in the source code, such as "Beijing Forbidden City", "Nanjing Forbidden City" and so on. Through analysis and processing, the text expression of concept node can be obtained. After the above processing, the final size of the concept nodes obtained is 5,813,824 nodes.

5. Processing of other information. Web page source codes have contained the entry summary information. The contents mainly include the definition and interpretation of the entry, etc. The synonymous relations, location relations and other resource management can be extracted through the language template. For example, "Taipei Forbidden City, formerly known as Zhongshan Museum, also known as National Palace Museum, located in Waishuangshi, Shilin District, Taipei City, Taiwan Province". The web page source code contains the attribute information of the entry and is presented in the form of an information box. Through analysis and processing, attribute relationship of the concept node can be obtained. The web page source code contains the description of the entry main body, and various types of relationship information of the concept nodes can be extracted by pattern clustering. Web page source tag contains open classification description information of the entry, and the open classification relationship of concept node can be obtained through analysis and processing. Through the above processing, the final 
scale of correlation relationship can be obtained as 19,995,579 relations, among which there are 2,514,567 undirected hyperedges.

News report web page information processing. There are a total of 1,980 news pages on "Syria's chemical weapon crisis in 2013", with the document size of about $10 \mathrm{MB}$.

1. Processing of concept nodes: By the concept node extraction method based on the combination of improved mutual information and information entropy, the size of concept node finally obtained is 1,082 nodes.

2. Processing of correlation relationship: The news corpus mainly reflects the cooccurrence relationship between concept nodes, and the size of the co-occurrence relationship finally obtained is 57,625 relations.

We construct the multimedia teaching resource management evaluation mainly on the information in 360 encyclopedia, and supplement it with the information extracted from the news report web pages, including 465 overlapped concept nodes and 26,829 overlapped relationships. The final scale of the multimedia teaching resource management evaluation nodes reaches 5,814,441 nodes, and the scale of correlation relationship reaches 20,026,375 relations. Figure 2 shows the teaching resource fragment diagram of the completed multimedia teaching resource management evaluation.

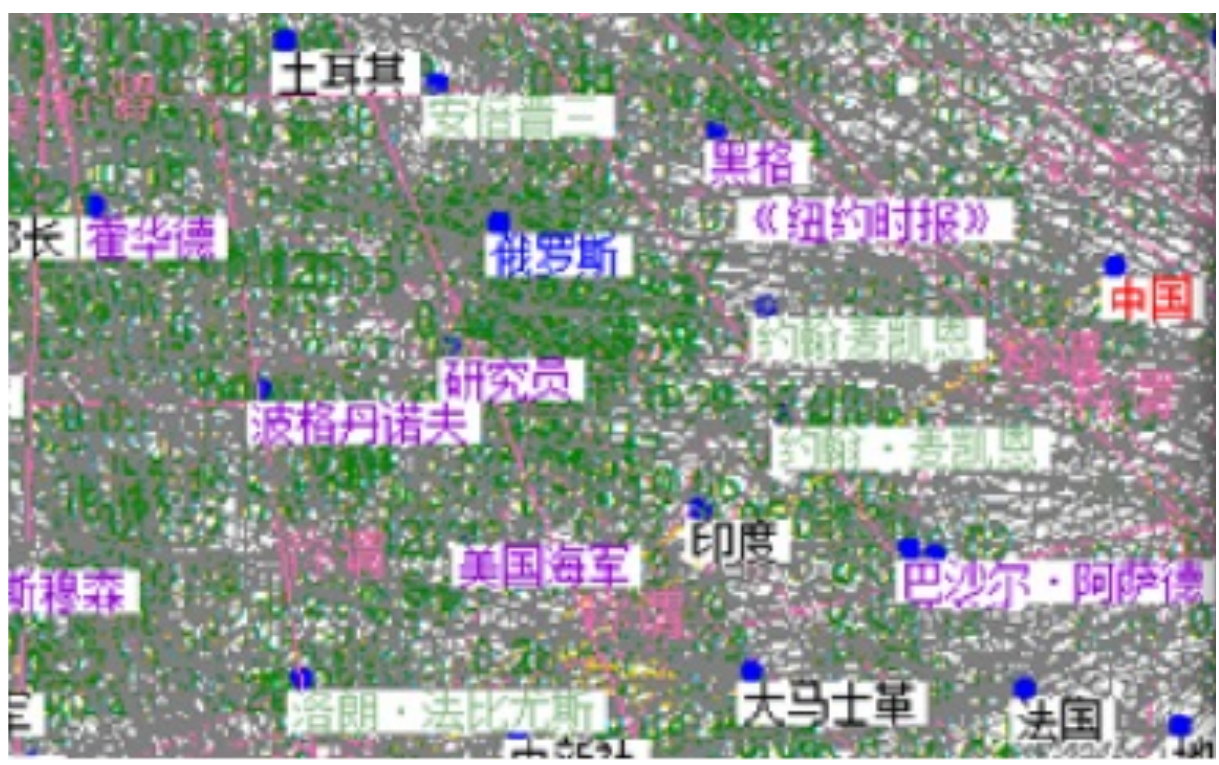

Fig. 2. Teaching resource fragment in the multimedia teaching resource management evaluation 


\subsection{Experiment Analysis}

In this paper, multimedia teaching resource management evaluation is constructed mainly based on Chinese data resources. The construction cost is small, the scale is relatively large, and the accuracy of resource management extracted is relatively high. Among them, the extraction of resource management for encyclopedia data has relatively high level of data structure. The extracted resource management is more targeted. And the accuracy rate is above $99 \%$ no matter in the concept node or deterministic relation extraction. In the extraction of ambiguous relationship, the relationship itself is used to handle the uncertainty of information, and its accuracy is mainly reflected in the accuracy of the concept node itself and therefore has relatively high degree of accuracy. In general, the multimedia teaching resource management evaluation resource management constructed in this paper has wide coverage, complex and diversified correlation relationships. The structure is open and flexible with very strong extensibility. It is application oriented and the resource management has timeliness. At the same time, the multimedia teaching resource management evaluation model based on hypergraph theory can handle uncertain and inaccurate information with relatively strong computability.

\section{Conclusions}

In this paper, multimedia teaching resource management evaluation model in the big data environment is proposed. The theoretical elements of the model and the evaluation method of the teaching resource management are expounded. Guided by the model and combined with the existing technologies, the construction method for the multimedia teaching resource management evaluation in the big data environment with open big data is studied. In addition, 360 encyclopedia and news corpus are used as teaching resources to construct the multimedia teaching resource management evaluation in the experiment.

\section{References}

[1] Zhou, L., Li, H., \& Sun, K. (2017). Teaching performance evaluation by means of a hierarchical multifactorial evaluation model based on type-2 fuzzy sets. Applied Intelligence, $1-11$.

[2] Kondo, A., Yamamoto, M., Inoue, Y., \& Ariyadasa, B. H. (2013). Evaluation of lead concentration by one-box type multimedia model in lake biwa-yodo river basin of japan. Chemosphere, 92(5), 497-503. https://doi.org/10.1016/j.chemosphere.2013.02.013

[3] Chaves, R. O., Wangenheim, C. G. V., Furtado, J. C. C., Oliveira, S. R. B., Santos, A., \& Favero, E. L. (2015). Experimental evaluation of a serious game for teaching software process modeling. IEEE Transactions on Education, 58(4), 289-296. https://oi.org/10.1109/ TE.2015.2411573

[4] Kohnle, A., Cassettari, D., Edwards, T. J., Ferguson, C., Gillies, A. D., \& Hooley, C. A., et al. (2012). A new multimedia resource for teaching quantum mechanics concepts. American Journal of Physics, 80(80), 148-153. https://doi.org/10.1119/1.3657800 
Paper-Construction of Multimedia Teaching Resource Management Evaluation Model in the Big ..

[5] Trestian, R., Ormond, O., \& Muntean, G. M. (2015). Performance evaluation of madmbased methods for network selection in a multimedia wireless environment. Wireless Networks, 21(5), 1-19. https://doi.org/10.1007/s11276-014-0882-z

[6] Gozalvez, J., Lucas-Estañ, M. C., \& Sanchez-Soriano, J. (2012). Joint radio resource management for heterogeneous wireless systems. Wireless Networks, 18(4), 443-455. https://doi.org/10.1007/s11276-011-0410-3

[7] Meixner, B. (2017). A pattern-based evaluation of download and cache management algorithms for annotated interactive non-linear videos. Multimedia Systems, 23(5), 527-561. https://doi.org/10.1007/s00530-016-0512-y

[8] Shimomura, T., \& Susono, H. (2012). Practice and evaluation of production of multimedia work in information education. Bulletin of the Chemical Society of Japan, 70(5), 10531060.

[9] Larson, M., Soleymani, M., Gravier, G., Ionescu, B., \& Jones, G. J. F. (2017). The benchmarking initiative for multimedia evaluation: mediaeval 2016. IEEE Multimedia, 24(1), 93-96. https://doi.org/10.1109/MMUL.2017.9

[10] [Kijne, J. W. (2011). Teaching irrigation science and water management: accepting professional diversity. editorial. Irrigation Science, 29(1), 1-10. https://doi.org/10.1007/s00271$\underline{010-0239-\mathrm{Z}}$

\section{$7 \quad$ Author}

Wei Bian is with The Academic Affairs Office of Xijing University, Xi'an, Shaanxi,China (bianwei@xijing.edu.cn).

Article submitted 07 February 2018. Final acceptance 23 February 2018. Final version published as submitted by the author. 